\title{
PARTICIPACIÓN SOCIAL, JUVENTUDES, Y REDES SOCIALES VIRTUALES: RUTAS
}

\section{TRANSITADAS, RUTAS POSIBLES}

\author{
YADIRA PALENZUELA FUNDORA ${ }^{1}$
}

\begin{abstract}
RESUMEN
El presente artículo problematiza el conocimiento producido sobre participación, jóvenes y redes sociales, en las investigaciones de la última década. Entre sus propósitos se encuentra comprender cómo se aborda la participación en el marco de las redes sociales virtuales, así como reflexionar qué sujeto juvenil se construye desde estas miradas, y qué efectos generan dichas aproximaciones. A partir del análisis de las rutas transitadas por la investigación social en esta temática, se identifican nuevos retos y esbozan otros caminos posibles, ofreciendo una definición de participación social que puede contribuir a enriquecer nuevos estudios sobre participación, jóvenes y redes sociales virtuales.
\end{abstract}

PAlabRas ClaVe: PARTICIPACIÓN SOCIAL, REDES SOCIALES VIRTUALES, JUVENTUDES

${ }^{1}$ Psicóloga y Máster en Psicología Educativa, Universidad de La Habana, Cuba. Magíster en Análisis Sistémico Aplicado a la Sociedad y Doctora en Psicología, Universidad de Chile. Miembro del Núcleo de investigación y acción en Juventudes FACSO y del Grupo de Prácticas Mediáticas, Instituto de Comunicación e Imagen (ICEI) de la Universidad de Chile. Correo electrónico: ypalenzuela@gmail.com. 


\title{
PARTICIPAÇÃO SOCIAL, JUVENTUDES, E REDES \\ SOCIAIS VIRTUAIS: ROTAS TRANSITADAS, ROTAS \\ POSSÍVEIS
}

\begin{abstract}
RESUMO
O presente artigo problematiza o conhecimento produzido sobre participação, juventude e redes sociais, nas pesquisas da última década. Entre seus propósitos está compreender como abordar a participação no contexto das redes sociais virtuais, bem como refletir sobre o sujeito juvenil construído a partir dessas perspectivas e quais são os efeitos que essas aproximações geram. A partir da análise das rotas transitadas pela pesquisa social nesta área, novos desafios são identificados e outras linhas possíveis são delineadas, oferecendo uma definição de participação social que pode contribuir para enriquecer novas pesquisas sobre participação, juventude e redes sociais virtuais.
\end{abstract}

PALAVRAS-CHAVE: PARTICIPAÇÃO SOCIAL, REDES SOCIAIS VIRTUAIS, JUVENTUDE.

\section{SOCIAL PARTICIPATION, YOUTHS, AND VIRTUAL SOCIAL NETWORKS: TRAVELED ROUTES, POSSIBLE ROUTES}

\begin{abstract}
The present article problematizes the knowledge obtained from research on participation, young people and social networks carried out in the last decade. It is purposed to understand, among others, how participation within the context of virtual social networks is approached, as well as to reflect on which juvenile subject is being constructed from these perspectives and the effects of such approximations. From the analysis of the already traveled paths by social research on this matter, new challenges are identified and other possible routes outlined in order to provide a definition of social participation, which can enrich new research on participation, youth and virtual social networks
\end{abstract}

KEYWORDS: SOCIAL PARTICIPATION, VIRTUAL SOCIAL NETWORKS, YOUTHS. 
5 Participación social, juventudes, y redes sociales virtuales: rutas transitadas, rutas posibles

\section{INTRODUCCIÓN}

EN LA ÚLTIMA DÉCADA, los estudios sobre la participación social de las/los jóvenes han adquirido gran relevancia para las Ciencias Sociales. El interés ha estado estrechamente vinculado a la emergencia de distintos movimientos sociales a nivel internacional, que posicionan con sus prácticas y formas de expresión, otras formas de participación social, que son observadas como nuevas, y donde el uso de las redes sociales virtuales ha estado presente ${ }^{2}$ (Corredor, Tirado e Iñiguez, 2010; Montenegro y Pujol, 2010; FernándezPlanells, Feixa y Figueras, 2013; Feixa, 2014; Subirat, 2011, 2015). Sin embargo, a pesar de estas evidencias, siguen siendo predominantes las investigaciones que entienden la participación social de forma tradicional donde el sistema electoral y la militancia en partidos políticos constituyen indicadores fundamentales para acreditar la las misma. Es así que, a través de diferentes informes de resultados de centros de estudios e instituciones interesadas sobre el tema, continúan legitimándose ideas como la baja participación, apatía, o desinterés de las personas jóvenes por la política y militancia en partidos (INJUV, 2010, 2013; INDH, 2015).

Por otra parte, en el año 2013, Chile era el país de la Organización para la Cooperación y Desarrollo Económico (OCDE) con mayor crecimiento en banda ancha y se encontraba dentro de los cinco países con mayor consumo de internet a través de la computadora y de los teléfonos inteligentes (SUBTEL, 2013). Dos años después, Chile ocupó el tercer puesto del ranking de esta organización en uso de redes sociales destacando por su presencia en Twitter

${ }^{2}$ Se utiliza el término virtual como sinónimo de aquellas acciones que ocurren en el escenario digital. No me adscribo a una división real/virtual de la realidad; o su homólogo offline/online como espacios contrapuestos o inconexos. Pues las prácticas que transcurren en el espacio "virtual" son tan reales como las que ocurren fuera de él. La perspectiva que asume este trabajo dará cuenta de lo anterior durante el desarrollo del mismo. 
(Gobierno de Chile, 2015) con acciones de gobierno electrónico entendidas estas como aquellas que se realizan para "facilitar el acceso, mediante el uso de tecnologías de información y comunicaciones, de los ciudadanos, organizaciones y gobierno a información, servicios y/o diálogo con la administración pública, a todos los niveles jerárquicos, organizacionales y territoriales" (Chateau, 2003, p.10). Además, en América Latina, Chile es uno de los países con mayor uso de redes sociales (96.7\%) solo siendo superado por México (98.3\%) y Argentina (97.6\%); consumiéndose alrededor de 17.6 horas online como promedio, de las cuales el $61.2 \%$ del tiempo lo consumen jóvenes menores de 35 años. (ComScore, 2014).

Este escenario nacional deviene en objeto de atención de diversas disciplinas de las Ciencias Sociales y las investigaciones sobre jóvenes y tecnologías en Chile cada vez más comienzan a incorporar en sus objetos de análisis, la temática de la participación en el ámbito digital (Arriagada y Schuster, 2008; Scherman, y Arriagada, 2010; Valenzuela, 2012; Palenzuela, 2013; Cabalín, 2014; Cárdenas, 2014; Aguilera, 2014), siendo un punto común en las aproximaciones el interés por conocer cómo se produce la participación juvenil en el entorno virtual, en especial en el contexto de los nuevos movimientos sociales que han tenido lugar en los últimos años.

Sin embargo, a pesar de que existe una amplia variedad de investigaciones que abordan esta problemática por el interés que ha adquirido a nivel mundial, muchas de ellas se centran en descripciones sobre los procesos de participación asociados a determinadas tecnologías o plataformas de redes sociales, en ocasiones sin problematizar a profundidad los propios conceptos que utilizan para observarlos (Cabello, 2014; Cárdenas, 2014). Es por ello, que resulta de interés problematizar el conocimiento producido sobre participación, redes sociales, y el lugar de las/los jóvenes en este escenario, sin pretender una definición acabada de las mismas; sino más bien con el objetivo de comprender cómo se ha constituido la participación social en la actualidad en estos 
7 Participación social, juventudes, y redes sociales virtuales: rutas transitadas, rutas posibles

escenarios, cómo funciona en los espacios de relaciones interpersonales mediados por las tecnologías; así como reflexionar qué sujeto juvenil se construye desde estas miradas, y qué efectos generan dichas aproximaciones. De esta forma, podremos construir las rutas transitadas por la investigación social en esta temática, problematizarlas, identificar nuevos restos y esbozar otras rutas posibles para investigar sobre participación, jóvenes y redes sociales virtuales.

\section{LOS ESTUDIOS SOBRE PARTICIPACIÓN SOCIAL Y REDES SOCIALES VIRTUALES EN LA ÚLTIMA DÉCADA}

\subsection{PARTICIPACIÓN Y REDES SOCIALES VIRTUALES: LA CONSTRUCCIÓN DEL SUJETO JUVENIL Y SU PARTICIPACIÓN EN ENTORNOS VIRTUALES}

Las investigaciones y producciones académicas contemporáneas que abordan la participación social en redes sociales virtuales de las/los sujetos jóvenes; no se presentan como un fenómeno homogéneo, ni se articulan desde una disciplina o campo de estudio particular. La mayoría de las investigaciones se desarrollan desde planteamientos y autores que dan cuenta de una mirada transdisciplinar, con distintas perspectivas teóricas que van desde las Ciencias Sociales (aportes de la sociología de la comunicación, antropología social, psicología social), las ciencias de la comunicación (estudios de recepción, teorías de consumos culturales, semiótica, etc.), hasta las ciencias físicas y matemáticas (teoría compleja de las redes y herramientas computacionales para las redes sociales). Esta gran diversidad de orientaciones teóricas para abordar la participación en un fenómeno reciente como lo son las redes sociales 
virtuales ${ }^{3}$ ha producido considerables artículos científicos en revistas indexadas de corriente principal (Thomson Reuters -ISI-, Scopus, Latindex, Scielo, etc.). La mayoría de estas investigaciones en los últimos años, se vinculan a la participación en los movimientos sociales que han sido objeto de estudio en distintas latitudes, destacándose las producciones sobre el Medio Oriente (Primavera Árabe); Estados Unidos (Occupy Wall Street) y en Iberoamérica, los casos de España (15 M) y Chile (Movimiento Estudiantil) por citar los más destacados. Debe tomarse en consideración, que la penetración, uso masivo y experiencias de participación en redes sociales virtuales, es un fenómeno social relevante cuyas manifestaciones datan de pocos años, por lo que constituye un tema novedoso y emergente (Montenegro y Pujol, 2010).

Por lo que si bien no existe una consolidada producción sobre la temática comparado con otros fenómenos que cuentan con mayor trayectoria de estudios; se puede realizar una sistematización de la última década, de acuerdo a los principales referentes conceptuales que utilizan, los énfasis que se realizan, los métodos que emplean; así como las construcciones sociales sobre los sujetos que participan y los efectos de estas aproximaciones.

En el presente texto se comprende la participación social de forma amplia (no solo vinculada a mecanismos formales e informales de participación política; sino también aquellas otras maneras de influir, ocupar y trasformar el espacio público), en temáticas relevantes para las/los sujetos que la conciben y llevan a cabo. Por ello, el abordaje que se realizará tomará en cuenta aquellas acciones que son constituidas y constituyentes de lo social en el terreno de lo político, y que son desarrolladas en el marco de las tecnologías (comprendidas

${ }^{3}$ Debe recordarse que el uso público y masivo de internet surge en los años 90, donde en 1996 se lograron conectar 10 millones de computadoras. Unos años después se ponen en circulación las principales redes sociales virtuales que hoy conocemos. Ej.: MySpace (2003); Hi5 (2003); LinkedIn (2003); Facebook (2004); Youtube (2005); Twitter (2006); Tumblr (2007); Foursquare (2009); WhatsApp (2009); Instagram (2010); Telegram (2013), etc. 
9 Participación social, juventudes, y redes sociales virtuales: rutas transitadas, rutas posibles

como producto de la interacción social y como productoras del espacio tecnosocial); específicamente en relación con las redes sociales virtuales -en tanto plataformas virtuales de relacionamiento interpersonal y de producción de contenidos materiales y simbólicos- (Montenegro y Pujol, 2010; Ardèvol et al., 2010; Subirat, 2011, 2015; Hine, 2004).

Teniendo en cuenta lo anterior, las producciones académicas sistematizadas y que devienen como rutas transitadas por la investigación social en esta temática, se pueden agrupar en tres grandes áreas de estudio: a) las que se focalizan en los tipos de participación y su vínculo con las redes sociales virtuales; b) las que remiten a las plataformas y usos de las mismas como espacios relevantes para las/los jóvenes; y c) aquellas que rescatan los significados y problematizan los efectos sociales y políticos que se genera en relación a estos espacios. Los tópicos que siguen dan cuenta de estas aproximaciones.

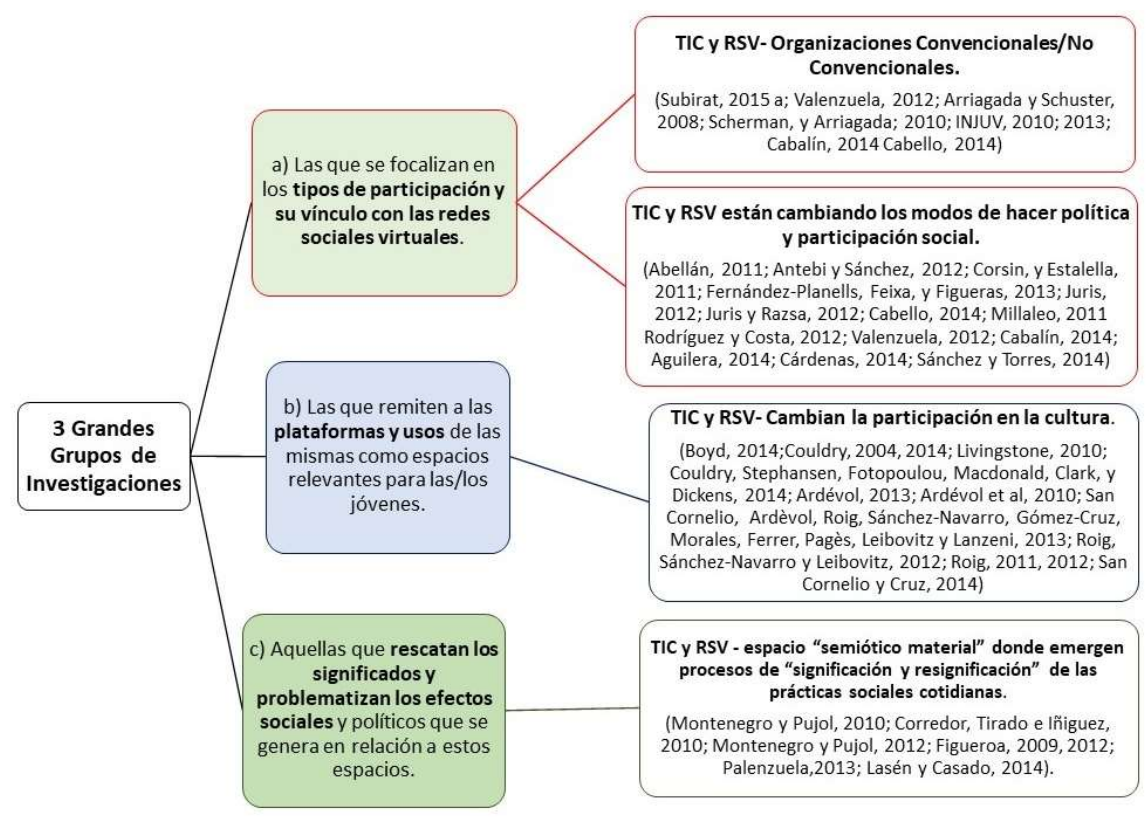

Figura 1. Áreas de investigación social sobre participación y redes sociales virtuales. Fuente: Elaboración propia. 


\subsection{RELEVANDO LAS PARTICIPACIONES JUVENILES}

Resulta común encontrar en los resultados de investigaciones actuales, ideas que describen a los jóvenes como entes pasivos, desinteresados por participar de la vida política y con alto grado de desconfianza y desmotivación. Estas ideas se sustentan en datos sobre la baja participación formal de las/los jóvenes en elecciones; la disminución de su adscripción a partidos políticos; la falta de confianza en las instituciones gubernamentales y el desagrado por tener cualquier vínculo formal con la esfera política y sus dirigentes; llegándose a describir a las/los jóvenes con fuertes calificativos como apáticos o alienados. (Morán y Benedicto, 2008; Arriagada y Schuster, 2008; Scherman y Arriagada; 2010; Valenzuela, 2012; INJUV, 2010, 2013). Sin embargo, por otra parte cada vez más aparecen evidencias de participación juvenil en el espacio público vinculados a causas sociales, ONG, o movimientos, donde las tecnologías y las redes sociales virtuales se hacen presentes (Montenegro y Pujol, 2010; Antebi y Sánchez, 2012; Corsin y Estalella, 2011; Fernández-Planells, Feixa y Figueras, 2013; Juris, 2012; Juris y Razsa, 2012; Torres y Costa, 2012; Cabalín, 2014; Aguilera, 2014; Cárdenas, 2014; Cabello, 2014; Sánchez y Torres, 2014).

Esta tensión se ve reflejada principalmente en investigaciones sobre participación sociopolítica de jóvenes en organizaciones políticas convencionales, ${ }^{4}$ en organizaciones políticas ${ }^{5}$ (Subirat, 2015, 2015a); así como

4 Subirat (2015) realiza una distinción entre las organizaciones políticas convencionales (OPC) y las organizaciones políticas no convencionales (OPNC). Las OPC las define como "aquellas que priorizan como forma de acción política la participación en espacios institucionales de toma de decisiones vinculadas a los procesos de creación de políticas públicas y lo hacen mediante procesos reglamentados de selección de participantes; por ejemplo, la participación electoral" (Subirat, 2015, p.76). 
11 Participación social, juventudes, y redes sociales virtuales: rutas transitadas, rutas posibles

en los novísimos movimientos sociales, ${ }^{6}$ que se distinguen de las anteriores porque funcionan en red, la utilización de las redes sociales virtuales es clave en su constitución, en la forma de operar y generar sus demandas; y tienen el potencial de pasar de una escala nacional a otra trasnacional con facilidad pues en esos espacios se mueven las/los jóvenes de hoy (Juris, Pereira y Feixa, 2012).

Es así que en este gran tópico de estudios que relevan las participaciones; se encuentran dos subgrupos o líneas de investigaciones. Una primera que agrupa aquellos estudios que caracterizan el uso que hacen de las TICs y redes virtuales las organizaciones sociales y políticas juveniles convencionales y no convencionales; tomando en su mayoría como conceptualización el término de participación ciudadana para dar cuenta de la participación que se realiza en relación con instituciones, organizaciones ciudadanas o grupos de asociatividad (Subirat, 2015a; Valenzuela, 2012; Arriagada y Schuster, 2008; Scherman y Arriagada, 2010; INJUV, 2010, 2013; Cabalín, 2014; Cabello, 2014). Otra segunda línea es aquella que agrupa los estudios que dan cuenta de cómo internet y las redes sociales están cambiando los modos de hacer política y de transformar la participación social. En este caso la misma se entiende como formas diversas de acción que se asocian con experiencias de participación en grandes movilizaciones y movimientos sociales -novísimos- que se gestan haciendo uso de las plataformas virtuales y que sus resultados apuntan a "cómo se están produciendo nuevas formas de organización colectiva" (Subirat, 2015a, p.6) a partir de modos de operar con

5 Son aquellas "nuevas formas de acción política" que se gestan bajo nuevos mecanismos y movimientos espontáneos y "que se articulan más allá — en una relación conflictiva - de las estructuras y cauces institucionales establecidos". (Subirat, 2015, p.128).

${ }^{6}$ Definición utilizada por Juris, Pereira y Feixa (2012) para referirse a los movimientos sociales contemporáneos "asociado con el surgimiento de nuevos modos de activismo colectivo en una era de redes globales y de ciberculturas juveniles" (Juris, Pereira y Feixa, 2012, p.25). 
las redes sociales. (Antebi y Sánchez, 2012; Corsin y Estalella, 2011; Fernández-Planells, Feixa y Figueras, 2013; Juris, 2012; Juris y Razsa, 2012; Cabello, 2014). En este segundo ámbito se destacan principalmente las producciones académicas españolas tanto de los equipos de investigación de la Universidad Autónoma de Barcelona, como de la Complutense de Madrid.

En Chile, los estudios de la primera línea se caracterizan por ser en su mayoría cuantitativos y emplear técnicas como cuestionarios y sondeos de opinión. Los resultados de algunas investigaciones de este tipo intentan demostrar, predecir o correlacionar ciertos comportamientos juveniles como lo son la participación en protestas callejeras según los usos que hacen de determinadas redes sociales ${ }^{7}$ (Valenzuela, 2012). Otros resultados dan cuenta de la relevancia que tienen estos espacios como nuevas formas de participación legítima por parte de las/los jóvenes, destacando su preferencia por las redes sociales como mejor herramienta de participación que otras tradicionales ${ }^{8}$ (INJUV, 2010, 2013; Arriagada y Schuster, 2008; Scherman, y Arriagada; 2010). Este tipo de investigaciones también destaca cómo las organizaciones políticas convencionales y no convencionales cada vez más incorporan las redes sociales en sus modos de operar -creando perfiles en páginas, posteando en las redes, convocando por Twitter o Facebook, creando eventos- para producir mayor cercanía con los sujetos que pertenecen a ellas, posicionar demandas y divulgar información sobre el trabajo que realizan (Cabalín, 2014). Desde esta

${ }^{7}$ En Chile, Valenzuela (2012) llega a esta conclusión con respecto a Facebook: "a mayor uso de estas plataformas, mayor fue la probabilidad de participar en las manifestaciones estudiantiles y políticas en la vía pública. De hecho, en 2011, el uso frecuente de Facebook fue la tercera variable más predictiva de participar en manifestaciones públicas" (Valenzuela, 2012, p.25).

${ }^{8}$ En Chile según el INJUV (2013) en su Séptima Encuesta Nacional de Juventud, el $61,4 \%$ de las y los jóvenes está de acuerdo con que "las redes sociales son una mejor herramienta que el voto para dar a conocer las demandas de la ciudadanía; el $69,4 \%$ considera que "sin redes sociales las manifestaciones serían mucho menos masivas en la actualidad" y el $41,4 \%$ refiere que "las redes sociales permiten incidir en forma directa en la toma de decisiones del estado" (INJUV, 2013, p.75). 
13 Participación social, juventudes, y redes sociales virtuales: rutas transitadas, rutas posibles

perspectiva la construcción juvenil que subyace a estos estudios ofrece una tendencia a observar a las/los jóvenes como consumidores de estas tecnologías, las cuales determinan o influyen en las formas de participación social que se realizan. De manera general, no existe una mirada crítica sobre el tema, pues esta se observa estrechamente vinculada a los medios de comunicación y redes sociales que se utilizan, en muchos casos sin conceptualizar a profundidad desde dónde se comprende y realizando un uso instrumental de la misma para describir la plataforma virtual a la que se hace referencia. (Cabalín, 2014; Cabello, 2014).

Por su parte, los estudios de la segunda línea que dan cuenta de cómo internet y las redes sociales están cambiando los modos de hacer política y de transformar la participación social, se caracterizan por conceptualizaciones amplias de participación en oposición a formas tradicionales de entenderlas acotadas solamente a la ciudadanía y la política. Metodológicamente emplean enfoques mixtos y algunos utilizan técnicas cuantitativas, de análisis de redes sociales (ARS) basadas en la ANT y/o teorías de Grafos (Fernández y Paniagua, 2012; Rodríguez, 2011; Congosto, 2015; Gualda, Borrero y Carpio, 2015), destacando cómo el uso de redes transforma la participación observando las interacciones que transcurren en estas redes. Esto lo evidencian dando seguimiento a las localizaciones, siguiendo \#hashtag, ${ }^{9}$ y conexiones significativas entre estos elementos para conocer pautas en la difusión de los movimientos y representar los principales nodos de participación. Otros estudios en esta línea, se centran en el análisis de casos, tomando a

${ }^{9}$ Es una etiqueta de metadatos precedida de un carácter especial que se utiliza en las redes sociales para que las plataformas y los usuarios identifiquen rápidamente los temas sobre los que se está conversando, facilitando la búsqueda y apelando a que aparezcan todas las conversaciones relacionadas con él. Pueden ser generadas por los propios usuarios para instalar un tema de conversación y cuando son las frases más repetidas en un momento concreto, se convierten en trending topic, lo que facilita mayor visualización y que se sumen nuevos usuarios. Ej.: \#NomásAFP; \#15M; \#AC (Asamblea Constituyente), \#NiUnaMenos; etc. 
determinados países, ciudades o eventos como casos de estudio para describir las características de los novísimos movimientos sociales y el papel que juegan las redes en ellos. Se destacan los estudios sobre el $15 \mathrm{M}$ o Movimiento de los Indignados en España (Antebi y Sánchez, 2012; Corsin y Estalella, 2011; Fernández-Planells, Feixa, y Figueras, 2013); Occupy Wall Street en EEUU (Juris, 2012; Juris y Razsa, 2012), la Primavera Árabe (Valdettaro, 2012; Carlón y Neto, 2012; González, 2014) o en el caso de Chile las experiencias de movimientos ambientalistas ${ }^{10}$ (Millaleo, 2011); del movimiento de estudiantes secundarios en el 2006 con el uso de páginas de fotologs que representaban a algún liceo u organización estudiantil (Rodríguez y Costa, 2012) y del Movimiento Estudiantil por la Educación, del 2011 (Valenzuela, 2012; Cabalín, 2014; Aguilera, 2014; Cárdenas, 2014; Sánchez y Torres, 2014). Estas investigaciones utilizan en su mayoría técnicas cualitativas de análisis de contenido en dichas plataformas, las que combinan con realización de entrevistas o análisis documental en medios de comunicación sobre el tema. Desde esta perspectiva la construcción social que se realiza de las/los sujetos jóvenes, destacan la agencia de los mismos, al realizar acciones de producción de eventos, convocatorias y resignificar los usos de las redes para su aplicación a las experiencias de movilización de las que son protagonistas. No obstante, en algunos de estos estudios se intenta resaltar el poder de las redes sociales en sí mismas y sus potencialidades como herramientas para la Revolución 2.0 (Valdetarro, 2012; Valenzuela, 2012), lo que produce efectos de invisibilizar y despolitizar la propia participación juvenil, evidenciándose en ocasiones cierto determinismo tecnológico (Cárdenas, 2014).

No obstante, si bien este es el ámbito que más se hace presente en las investigaciones de la última década sobre el tema, también se destacan otros

10 El caso de Punta Choros y Barrancones sobre la construcción de centrales termoeléctricas en el 2011 Ver: http:/www.emol.com/noticias/tecnologia/2010/08/25/432526/punta-de-choroschilenos-acuden-a-las-redes-sociales-para-manifestar-su-descontento.html 
15 Participación social, juventudes, y redes sociales virtuales: rutas transitadas, rutas posibles

estudios que ponen el foco en las plataformas y los usos participativos que se le dan a las mismas por parte de las/los sujetos jóvenes. A continuación, se presentan algunas investigaciones de referencia al respecto.

\subsection{RELEVANDO LAS PLATAFORMAS Y USOS DE LAS REDES SOCIALES COMO ESPACIOS DE PARTICIPACIÓN Y PRODUCCIÓN PARA LAS/LOS JÓVENES}

En la última década, aparecen un conjunto de producciones académicas que abordan la participación en los nuevos medias y redes sociales virtuales de las/los sujetos jóvenes; relevando los usos, apropiaciones y prácticas participativas que se desarrollan en estas plataformas. Si bien estas aproximaciones tienen menor presencia en comparación con lo tratado anteriormente. Resultan interesantes destacar algunas de ellas.

Desde esta mirada, el centro del análisis se posiciona en las plataformas y redes sociales, así como en los consumos culturales ${ }^{11}$ y prácticas que gestan en ellas las/los jóvenes. Las tecnologías y las redes sociales virtuales son entendidas entonces como herramientas que transforman los modos en que estos sujetos participan en la cultura. A decir de Reguillo (2007):

"Para intentar comprender los sentidos que animan a los colectivos juveniles y a los jóvenes en general, hay que desplazar la mirada de lo normativo, institucionalizado y del "deber ser", hacia el terreno de lo incorporado y lo actuado: buscando que el eje de "lectura" sea el propio

${ }^{11}$ Según Martín Barbero (1987) los consumos culturales deben entenderse como "producción de sentidos: lugar de una lucha que no se agota en la posesión de los objetos, pues pasa aún más decisivamente por los usos que les dan forma social y en los que se inscriben demandas y dispositivos de acción que provienen de diferentes competencias culturales (...) donde cobran importancia las luchas contra las formas de poder que atraviesan (...), la vida cotidiana y las luchas por la apropiación de bienes y servicios" (Martín Barbero, 1987, p. 235). 
joven que, a partir de las múltiples mediaciones que lo configuran como actor social, "haga hablar" a la institucionalidad" (Reguillo, 2007, p. 69)

Es así que surgen en este ámbito un grupo de producciones académicas fundamentalmente desde EEUU (Boyd, 2014), Inglaterra (Couldry, 2004; Couldry, 2014; Livingstone, 2010; Couldry, et al, 2014) y España (Ardèvol, 2013; Ardèvol et al., 2010; San Cornelio et al, 2013; Roig, Sánchez-Navarro y Leibovitz, 2012; Roig, 2011; San Cornelio y Cruz, 2014) que dan cuenta de cómo las tecnologías, las redes sociales y las prácticas que se gestan en torno a ellas; están potenciando la emergencia de nuevos sujetos juveniles, donde la participación cobra matices particulares.

Algunas investigaciones consideran que las redes sociales virtuales como plataformas de relacionamiento en internet, constituyen un lugar donde las/los adolescentes socializan y participan por excelencia no solo en la política sino también en otras esferas de su cotidianidad. Esto porque en la actualidad se encuentran restringidos para ellos, otros espacios públicos en comparación con generaciones anteriores. En este sentido las redes sociales devienen en espacios idóneos de encuentro e interacción con los otros, por lo que emergen como el espacio público por excelencia donde socializar y participar (Boyd, 2014).

Por su parte investigadores ingleses, también orientan sus producciones académicas a destacar el potencial de estas herramientas en pos de la participación por las prácticas mediáticas que se erigen desde las mismas, las cuales contribuyen a ampliar las escalas de interacciones más allá de la puramente local (Couldry, et al., 2014). Sin embargo algunos de los miembros de estos equipos se muestran muchos más críticos con las afirmaciones que le otorgan el poder de la participación contemporánea a estas plataformas, pues reconocen que si bien permiten una movilización política más rápida en el corto plazo, nuevas formas de acción colectiva y ciclos acelerados de acción; en el largo plazo no se observan contribuciones mayores como para sustentar el 
17 Participación social, juventudes, y redes sociales virtuales: rutas transitadas, rutas posibles

argumento de que se puede gestar cambio político y transformación desde ellas. Es así que minimizan el papel de las mismas para la acción política y la transformación en el largo plazo; llegando abiertamente a expresar que son un mito pues generan una idea del nosotros y de lo colectivo que está intencionado, y forma parte del propio valor económico de estas plataformas. (Couldry, 2014). Otras investigaciones en esta área, si bien destacan la importancia de la participación a partir de estudios que triangulan técnicas cuantitativas y cualitativas al trabajar en Reino Unido con adolescentes y sus padres; obtienen como resultados que solo cuando las estructuras institucionales -familia, escuela, organizaciones, compañeros- apoyan y potencian la participación cívica de las/los jóvenes; es que se observa participación en la esfera pública ya sea online u offline. (Livingstone, 2010). Estos estudios concluyen que a pesar de que se reconoce que con las redes sociales virtuales, se dan las condiciones para que las/los adolescentes participen por excelencia, esta participación se pone en juego con los otros y en ocasiones los sujetos refieren que "no sienten que se les escucha ni toman en cuenta" aunque participen por esta vía (Livingstone, 2010, p.15).

Por otra parte, y contrastando lo anterior, los investigadores españoles son más optimistas, y retoman la idea de la centralidad de las redes sociales como lugares donde no solo se participa más, sino que se expresan nuevas formas de participación juvenil que no eran observadas con anterioridad. En este caso las investigaciones tampoco se centran en el marco de la acción política, sino que conceptualizan la participación en sentido más amplio, como nuevas formas de accionar y transformar la vida cultural y social en la actualidad. Sus estudios se focalizan en la generación y producción de contenidos culturales y cómo las nuevas formas de participación desde este ámbito contribuyen a cambiar las relaciones de poder que se hacen presentes en la cotidianidad. (Ardèvol, 2013; San Cornelio et al., 2013; Roig, 2011). 
San Cornelio et al. (2013) definen la participación en estos nuevos medias y redes sociales como un fenómeno cultural que afecta a todos y que se debe asumir en la actualidad como "una fuerza cultural que empuja (...) toma diferentes voces y diferentes articulaciones" (San Cornelio, et al., 2013, p.67). Estos autores diferencian este modo de entender la participación en el ámbito cultural, de aquellas otras formas ideológicas, desde las que tradicionalmente se ha comprendido la participación. De esta manera considero que se centran en formas prácticas de operar ante las exigencias del mercado y la sociedad, pues según su opinión necesariamente los nuevos tiempos exigen instancias de participación en todas partes. Estas producciones académicas por lo general trabajan con participación de las/los jóvenes en la creación de productos culturales y es en este sentido que consideran que existen en la actualidad nuevas formas de participación social; entre las que se destacan: el crowdfunding ${ }^{12}$ y la co-creation; ${ }^{13}$ como evidencia de "nuevas agencias culturales que ponen en crisis la división entre productores y públicos" (Ardèvol, 2013, p.8).

Lo que guardan en común la mayoría de las producciones de este apartado en lo metodológico es un esfuerzo por la utilización de técnicas cualitativas (entrevistas a profundidad, grupos de conversación, etnografías, entre otros), para abordar las prácticas participativas en estas plataformas virtuales. Lo que me llama la atención en la mayoría de estos estudios que ponen el foco en las experiencias de participación en el contexto de la

${ }^{12}$ Se refiere a la estrategia de participación y cooperación colectiva llevada a cabo por individuos que conforman una red con el objetivo de conseguir dinero u otros recursos. En el caso de las investigaciones se refieren al crowdfunding audiovisual como alternativa participativa para encontrar los recursos y hacer una película donde participan todos y de esta forma "controlar el resultado, no sometido a las leyes del mercado -aunque sí a las de la comunidad y a las reglas pactadas" (San Cornelio et al, 2013, p. 66)

${ }^{13}$ Se refiere a "una participación explícita de usuarios" que contribuyen a la realización conjunta de un producto cultural, formando parte de comunidades de desarrollo que existen en internet y las redes sociales. (San Cornelio et al, 2013, p. 52) 
19 Participación social, juventudes, y redes sociales virtuales: rutas transitadas, rutas posibles

producción cultural y cómo cambian las formas de participar en la sociedad en general, es que sus investigaciones no abordan la problemática de la acción colectiva en el terreno de lo político. Estos vacíos dejan abierta la interrogante por las posibilidades de articulación de los contenidos materiales y simbólicos culturales en el marco de la acción colectiva y si en estos escenarios también cambian o se transforman las formas de participación de las/los jóvenes. Es así que si bien estos estudios construyen un/a sujeto juvenil que se destaca por su agencia en tanto producen, construyen y transforman las prácticas culturales en la actualidad; estos nuevos agenciamientos juveniles no son abordados suficientemente en el marco la acción colectiva, constituyendo un escenario posible para llevar a cabo nuevas investigaciones en esta línea.

Muchas de las críticas realizadas hasta acá, son tomadas en cuenta por un número menor de investigaciones que conforman a partir de la sistematización realizada, el tercer ámbito de estudios. Estos ofrecen pistas para trazar nuevas rutas en los futuros estudios sobre participación social y redes sociales virtuales de sujetos jóvenes. En este último apartado se reúnen los estudios que relevan el papel de los significados y problematizan los efectos sociales y políticos de las tecnologías en estos nuevos escenarios del espacio público.

\section{RESCATANDO LOS SIGNIFICADOS Y PROBLEMATIZANDO LOS EFECTOS SOCIALES Y POLÍTICOS DE LA PARTICIPACIÓN EN REDES SOCIALES VIRTUALES}

En los últimos años entre las investigaciones que abordan la participación de las/los jóvenes en internet, redes sociales y usos de dispositivos tecnológicos en su vida cotidiana -ej.: teléfonos móviles que les permiten hacer y tener todo eso encima, como parte de su propio cuerpo (Figueroa, 2012)-; se 
encuentran algunas publicaciones académicas que destacan la necesidad de que los estudios de Ciencias Sociales y tecnologías, pongan el énfasis en las significaciones de las prácticas sociales relacionadas con ello; así como en problematizar sobre los efectos y construcciones que subyacen a los conocimientos que se producen en esta área. (Corredor, Tirado e Iñiguez, 2010; Montenegro y Pujol, 2010; Figueroa, 2012; Lasén y Casado, 2014).

Estas miradas críticas provenientes fundamentalmente desde la psicología social, si bien son minoritarias en el escenario de las investigaciones sociales sobre tecnologías; emergen como producciones académicas potentes que se resisten a visiones hegemónicas que le otorgan valor al poder de las tecnologías en sí mismas, ya sea desde concepciones ciberoptimistas o ciberpesimistas (Montenegro y Pujol, 2010; Figueroa, 2012). Es así que surgen no solo como resistencia realizando críticas a estos estudios convencionales que destacan ventajas o riesgos de las tecnologías y redes sociales para los individuos en la sociedad contemporánea; sino que también ofrecen alternativas y nuevas preguntas para develar los vacíos o ausencias en investigaciones sobre la temática. De esta forma instalan nuevas perspectivas de análisis: la cuestión de los mecanismos de control social que están presentes, la normalización, resistencia, panoptismo, relaciones de poder invisibilizadas en el uso de los dispositivos y las redes (Corredor, Tirado e Iñiguez, 2010); las naturalizaciones en las formas de entender las tecnologías, los juicios morales y concepciones tradicionales que se utilizan para comprender fenómenos emergentes (Figueroa, 2012); la posibilidad de replantearse otros modos de construir la juventud y el género desde posturas ciberactivistas y ciberfeministas (Montenegro y Pujol, 2010); o la necesidad de pensar nuevas estrategias académicas de estudios para comprender los nuevos modos y "dinámicas de organización social, la conformación de nuevos híbridos sociotécnicos al servicio de lo político y de la praxis productiva y creadora que resulta ser la acción colectiva" (Cabello, 2014, p.13). 
21 Participación social, juventudes, y redes sociales virtuales: rutas transitadas, rutas posibles

Estos estudios que apuestan por una mirada que releve las significaciones y problematice críticamente los efectos sociales y políticos que se generan; asumen además un ejercicio reflexivo en tanto auto observan y visibilizan sus propósitos, explicitando en sus propuestas caminos o rutas teóricas y metodológicas que emplean para intentar develar o subvertir las relaciones de poder que se ponen en juego desde la propia producción del conocimiento, y cómo las mismas responden a lógicas que contribuyen o no a la reproducción o transformación de la sociedad. (Montenegro y Pujo, 2010; Corredor, Tirado e Iñiguez, 2010; Figueroa, 2012; Lasén y Casado, 2014).

De esta forma metodológicamente apuestan por enfoques cualitativos que articulan técnicas de análisis crítico del discurso, grupos de discusión y descripciones densas (Corredor, Tirado e Iñiguez, 2010); etnografías, entrevistas a profundidad que ponen en diálogo con datos secundarios (Figueroa, 2012; Lasén y Casado, 2014; Cabello, 2014); estudios de casos y experiencias de investigación-acción que se analizan y reflexionan desde paradigmas críticos y ciberfeministas (Montenegro y Pujol, 2010); o técnicas de análisis del discurso desde una perspectiva interdisciplinar que combina aportes de la semiótica social y la ciencia cognitiva y dialoga con análisis documental y etnografía virtual (Cárdena, 2014).

Por último, a diferencia de los dos apartados abordados, este grupo de investigaciones posiciona sus resultados desde una perspectiva situada, haciendo alusión a que son fenómenos cambiantes no solo por lo vertiginoso del cambio social, sino también por la propia dinámica de los fenómenos que se intentan comprender por lo que exige una aproximación siempre incompleta y sin pretensiones de generalización, ni de posicionamientos como saberesverdad. (Montenegro y Pujol, 2010; Figueroa, 2012; Ibáñez, 2001). Por lo que si bien no tratan directamente la participación social en redes sociales virtuales de las/los sujetos jóvenes -aunque hacen referencia en alguna medida a ello-; a través de sus diferentes énfasis y propuestas, se logran rescatar una serie de 
pistas a tomar en cuenta para trazar nuevas rutas investigativas sobre la problemática.

\section{RUTAS POSIBLES}

Teniendo en cuenta las rutas transitadas por las producciones académicas de la última década, sobre la problemática de la participación, jóvenes y redes sociales virtuales; se evidencia que las elaboraciones de sentido y construcciones sobre el tema se debaten en un escenario heterogéneo, donde no existe una única forma de entender el fenómeno; sino que el mismo se construye a partir de elementos que se le atribuyen y énfasis que se relevan en determinados contextos socioculturales, políticos, geográficos e históricamente situados. El mapa de rutas transitadas que destaca los tres ámbitos de estudios realizados (que también dan cuenta de mi posicionamiento y forma de contribuir a construir esa realidad, pues la organización pudiera haber sido otra); apuntan a su vez a problematizar los distintos matices, y opciones epistemológicas, teóricas y políticas de los autores que contribuyen a legitimar o subvertir dichas producciones de sentidos en el escenario de la investigación social

En esta diversidad de perspectivas y propuestas que pueden darse sobre la participación social en el marco de las nuevas tecnologías, y específicamente en el escenario de las redes sociales virtuales, me arriesgo a comenzar a esbozar una ruta alternativa que rescate los significados y problematizan los efectos sociales y políticos que se genera en relación a estos espacios. Bajo estas coordenadas y tomando como brújula epistemológica el construccionismo social (Gergen, 2006; Ibáñez, 2001, 2004; Iñiguez, 2003), y como sustento teórico la psicología social crítica (Piper, 2002; Ibáñez, 2001, 2004; Iñiguez, 2003; Montenegro y Pujol, 2010, 2012; Corredor, Tirado e Iñiguez, 2010; 
23 Participación social, juventudes, y redes sociales virtuales: rutas transitadas, rutas posibles

Figueroa, 2012, 2017) en diálogo con aproximaciones estéticas de la disciplina (Fernández, 1999, 2010); y otras ramas de las ciencias sociales (Reguillo, 2007, 2016, 2017; Jacques Rancière, 2006, 2009; Duarte, 2015); comparto lo que comprendo por participación social en el escenario de las redes sociales virtuales.

De esta manera entiendo la participación social de manera amplia como 'forma' de acción colectiva en el terreno de 'lo político'. Lo significa que no solo estará vinculada a mecanismos y prácticas formales e informales de participación política; sino también a aquellas otras prácticas y maneras de influir, ocupar, producir y trasformar el espacio público -físico y/o virtual-, en temáticas relevantes para las/los sujetos bajo distintos formatos, modos de organización, asociatividad o movimientos; destacando el carácter simbólico y afectivo de las mismas y reconociendo la multiplicidad de códigos e instancias de expresión significadas por sus actuantes.

Esta opción de asumir la participación y apellidarla de social, tiene el objetivo de dotar de sentido y resignificar la categoría social, muchas veces relegada y contrastada con la categoría política cuando de participación se habla. Tomar esta decisión, en parte refleja la necesidad de ajustar las conceptualizaciones que utilizamos cuando observamos y comprendemos problemáticas juveniles al hacer y decir de las/los propios jóvenes; quienes en mi experiencia empírica no se identifican con otras formas de enunciación como participación ciudadana por la relación con el Estado que implica; o participación política por la relación con organizaciones, instituciones o espacios formales de participación, que remiten en alguna medida al sistema político (Palenzuela, 2013).

Definir participación social como categoría amplia, inclusiva, que acoge diversas formas y expresiones de acción colectiva en escenarios presenciales y/o digitales; no necesariamente se contrapone a lo político. En especial si se 
entiende lo político como momento instituyente, sin contenido prefijado, potenciador de acontecimiento social como espacio simbólico y de disenso en contraposición a la política tradicional; la que remite a la lógica instrumental de administración de lo instituido, a las formalidades e instituciones del sistema político que conocemos (Mouffe 2007; Retamozo, 2009).

Avanzar en esta dirección, implica a su vez una comprensión particular de las/los jóvenes más allá de ser entendidos por su categoría etaria o elementos biológicos y psicológicos. Por lo que me identifico con la propuesta de Duarte (2015) denominada "construcción social de juventud" en la cual se asume:

como un proceso cuyas características más significativas están dadas por la inscripción social, política, económica y cultural de experiencias, en que cada formación socio-histórica define como juventud y lo juvenil. De esta forma, cuestiones como la clase social de pertenencia, el género, el origen racial, la localización territorial, la adscripción (sub o contra) cultural, entre otros, tienen un peso significativo en su conformación de identidad y en sus experiencias como jóvenes. (Duarte, 2015, p.21)

Asumir esta perspectiva de análisis contribuye a la construcción de un sujeto juvenil que se constituye y es constituido en las propias interacciones que establece en el entramado de sus relaciones sociales cotidianas. Destaca también un posicionamiento y capacidad de agencia en tanto producen, construyen y transforman las prácticas culturales en la actualidad, visibilizando nuevas formas de expresión de la participación y transformación social en el contexto de la acción colectiva. Además, implica asumir el reto de develar los patrones adultocéntricos que subyacen en las construcciones académicas que visualiza a las/los jóvenes como entes pasivos que no participan o que lo hacen condicionados por las nuevas tecnologías y las redes sociales.

Comprender la participación social como forma de acción colectiva, desde una aproximación estética, implica visibilizar otras maneras de construir 
25 Participación social, juventudes, y redes sociales virtuales: rutas transitadas, rutas posibles

el mundo de las prácticas políticas juveniles como evidencias sensibles que al mismo tiempo hace visible la existencia de un común distribuido y repartido, al cual tensionan y resisten produciendo otras formas posibles (Rancière, 2006, 2009). Además, es coherente con la propuesta comprensiva que se asume para entender las juventudes y relevar las experiencias que ponen en juego desde las distintas inscripciones a las que pertenecen en el contexto de la sociedad contemporánea (Duarte, 2015).

A modo de síntesis, puedo expresar que entender la participación social como 'forma' de acción colectiva en el terreno de 'lo político'; supone centrar la atención en el reconocimiento de diversas maneras de sociabilidad -nuevos escenarios de interacción y organización físico/digital-; así como reconocer otros modos posibles de socialidad -nuevas estrategias y códigos comunicacionales para hacerlas posibles/inteligibles en estos espacios donde la afectividad tiene un lugar central-. Este esfuerzo estético que implica activar otras sensibilidades en los análisis, se propone a su vez, romper con el mundo común que se ha instalado en el campo de las ciencias sociales con respecto a la investigación sobre participación, jóvenes e internet.

Definiciones normativas sobre la problemática, clasificaciones y tipologías de participación como categorías cerradas, o realizar descripciones poco situadas; contradicen la propuesta que aquí realizo. Por otra parte, aproximarse a estos estudios sin hacernos la pregunta por el compromiso político sobre los conocimientos que producimos, puede contribuir a validar desde juicios de valor, definiciones, maneras y espacios de participación que remiten a calificativos como buena/mala, correcta/incorrecta, aceptable/inaceptable, etc. que tributan a seguir legitimando el mundo instituido de formulaciones sobre la problemática (Reguillo, 2017).

Dar cuenta de otras sensibilidades posibles sobre la participación social en el ámbito de la vida cotidiana no es una tarea menor, conlleva asumir el 
compromiso e invitación de otros autores a arriesgarse y cambiar las preguntas que nos hacemos (Reguillo, 2017), innovar teórica y metodológicamente en el campo de la Psicología, que como disciplina a pesar de sus potencialidades, ha estado rezagada en esta labor (Figueroa 2012; 2017) y mejor aún no cansarse o aburrirse en este intento de devolver la estética y estudio de la sensibilidad y las emociones a la psicología social (Fernández, 1999); especialmente en temáticas de larga trayectoria investigativa como es la participación social.

Algunas formas de comenzar a trabajar en estos retos serían ofrecer a las/los propios jóvenes la oportunidad de autodefinir lo que es para ella/os participación, permitir que den cuenta de todas las formas y modos de expresión en los que consideran que participan socialmente. Esto enriquecerá sin dudas las producciones académicas y ampliará los escenarios posibles de estudios. Otra clave no menos importante implicará observar sistemáticamente, tensionar, poner en discusión, tomar en cuenta aquellas acciones colectivas que son constituidas y constituyentes de lo social en el terreno de lo político, y que son desarrolladas en el marco de las tecnologías, comprendidas como producto de la interacción social y como productoras del espacio tecnosocial (Montenegro y Pujol, 2010).

Transitar por esta ruta que propongo que pone el énfasis en los significados y problematiza los efectos sociales y políticos de estas aproximaciones; implica reconocer a las redes sociales más que como plataformas virtuales de uso cotidiano (ej.: Facebook, Twitter, WhatsApp, etc.) como espacios de producción de contenidos materiales y simbólicos que constituyen narraciones de nuestra cotidianidad. Esto nos remite a una comprensión de las tecnologías como construcción social, destacando la simetría y relevancia que tienen dichas plataformas y los dispositivos en los que se alojan, en la construcción social que se realiza. Por lo que una comprensión compleja del fenómeno de la participación en las redes sociales virtuales, debe analizarse de forma situada, dando cuenta del contexto histórico cultural donde 
27 Participación social, juventudes, y redes sociales virtuales: rutas transitadas, rutas posibles

estas se gestan y dotan de sentido. (Reguillo, 2017; Figueroa, 2017; Montenegro y Pujol, 2010; Ardèvol et al., 2010; Subirat, 2015; Hine, 2004).

Un elemento de interés para recorrer este camino, lo constituye su abordaje desde una perspectiva estética. El énfasis estético en el marco de un entendimiento de la realidad social desde el construccionismo social, implica ir más allá de los discursos y sus efectos; para comprender la 'forma' de la participación social como afectividad. Entender la participación social como afectividad, posibilita además de ofrecer comprensiones críticas o develar las prácticas sociales que contribuye a legitimar, reforzar o subvertir; que se oriente también como una estrategia que busque 'dar sentido' a la realidad, desde otros lugares posibles. Esta mirada que involucra el diálogo de las dos psicologías de lo social (Fernández, 2005), se centra en la búsqueda de esta sensibilidad en lo cotidiano de la participación, que en ocasiones no se presenta en palabras, lo que implica un esfuerzo por dar cuenta de esas otras formas de expresión de lo sensible sin que esto implique una separación o un tratamiento escindido entre ambas cosas. Sin dudas, lograr articular lo anterior es todo un reto, pero no un imposible porque como bien dice Fernández (1999): “en efecto, ni las formas ni los afectos están separados en sentidos de la percepción, porque son una entidad estética unitaria, homogénea, indistinta" (p. 88).

Incorporar lo afectivo en la comprensión de la participación social, requiere obviar la búsqueda de una lógica o evitar describir los mecanismos por los cuales esta se presenta, pues "afectivo será pues todo aquello que no tiene lógica ni tiene pensamiento, pero tiene realidad y tiene forma" (Fernández, 1999, p. 152), y la manera de acceder a ello es a través de la estética que es el sentido que "unifica, hace congruente, da razón de ser y sirve de guía" (Fernández, 2010, p.6); y para lograrlo siguiendo a este autor, hay que sentir la realidad social como viva, es necesario estar dentro. 


\section{REFERENCIAS BIBLIOGRÁFICAS}

Aguilera, O. (2014). Generaciones: movimientos juveniles, políticas de la identidad y disputas por la visibilidad en el Chile neoliberal. Colección Becas de Investigación CLACSO. Buenos Aires. ISBN 978-987-722032-2.

Antebi, A. y Sánchez, J. (2012). Plazas fuertes: de Midan Tahrir a la Plaça de Catalunya, espacio público y revueltas populares contemporáneas. En M. Urban Crespo (coordinador): ¡Ocupad el mundo! Barcelona: Editorial Icaria Antrazyt.

Ardèvol, E. (2013). Cultura digital y prácticas creativas. Tientos etnográficos en torno a la Cultura Libre. IN3 Working series paper, pp. 1-47.

Ardèvol, E., Gómez-Cruz, E., Roig, A. y San Cornelio, G. (2010). Prácticas creativas y participación en los nuevos media. Quaderns del CAC, 34, 13(1), pp. $27-38$.

Arriagada, A. y Schuster, M. (2008). Consumo de medios de comunicación y participación ciudadana de los jóvenes chilenos. Cuaderno de información. No 22, 2008-1. ISSN0716-162X. pp. 34-41. Recuperado de http://www.cuadernos.info/index.php/CDI/article/view/87/79

Boyd, D. (2014). It's complicated: the social lives of networked teens. New Haven; Yale Universit Press. London.

Cabalín, C. (2014). Estudiantes conectados y movilizados: El uso de Facebook en las protestas estudiantiles en Chile. Revista Comunicar. $\mathrm{N}^{\mathrm{o}} 43$, v. XXII, ISSN: 1134-3478

Cabello, P. (2014). Organizaciones ciudadanas en la sociedad del conocimiento y la información: nuevos medios, nuevas formas de participación. [Editorial]. Revista Colombiana de Ciencias Sociales, 5(1), pp. 13-17.

Cárdenas, C. (2014). Inútiles y subversivos: representación transmedia de los estudiantes chilenos en redes sociales. Romanica Olomucensia 2014 vol. 26 no. 2.

Carlón, M; Neto, F. (comp.) (2012). La política de los internautas: Nuevas formas de participación. Buenos Aires: Editorial La Crujía.

Chateau, J. (2003) (coord.). Gobierno electrónico en Chile: Estado del Arte. Proyecto de Reforma y Modernización del Estado Ministerio Secretaría General de la Presidencia y Programa de Modernización de la Gestión Pública. Departamento de Ingeniería Industrial - Universidad de Chile. Ediciones Praxis y Ciencia Política. Recuperado de: http://www.ederecho.cl/downloads/gobiernoelectronicoenchilepdf.pdf 
29 Participación social, juventudes, y redes sociales virtuales: rutas transitadas, rutas posibles

Congosto, M. (2015). Elecciones Europeas 2014: Viralidad de los mensajes en Twitter. REDES- Revista hispana para el análisis de redes sociales. Vol.26, No.1. Universidad Autónoma de Barcelona. España. ISSN 1579-0185

Corsin, J. y Estalella, A. (2011). \#spanishrevolu-tion. Anthropology Today $\mathrm{N}^{\circ} 27(4)$.

Corredor, F, Tirado, F. e Iñiguez, L. (2010). ¿Bajo las riendas del teléfono móvil? Control social, normalización y resistencia. Psicología \& Sociedad, 22 (1) pp. 60-69.

Couldry, N., Stephansen, H., Fotopoulou, A. Macdonald, R., Clark, W. y Dickens, L. (2014). Digital citizenship? Narrative exchange and the changing terms of civic culture. Citizenship Studies, 18 (6-7). 615-629. ISSN 1362-1025

Couldry, N. (2014). The myth of 'us': digital networks, political change and the production of collectivity Information. Communication \& Society. 1-38. ISSN 1369-118X

Couldry, N. (2004). Theorising Media as Practice. Social Semiotics, 14 (2), pp. $115-132$.

Duarte, K. (2015). El adultocentrismo como paradigma y sistema de dominio. Análisis de la reproducción de imaginarios en la investigación social chilena sobre lo juvenil. Memoria para optar al grado de Doctor en sociología. Recuperado de: https://www.educacion.gob.es/teseo/imprimirFicheroTesis.do?idFichero $=60938$

Feixa, C. (2014). De la generación@ a la \#generación. La juventud en la era digital. Barcelona: NED Ediciones.

Fernández, M. y Paniagua, F. (2012). El poder de las redes sociales en la política y en los movimientos sociales. En Cotarelo, R.; Crespo, I. La comunicación política y las nuevas tecnologías. España: Catarata, pp.130-150. ISBN 978-84-8319-773-8.

Fernández, P. (2010). Introducción o la razón estética. Universidad Nacional Autónoma de México.

Fernández, P. (2005). Los dos lenguajes de las dos psicologías de lo social. Athenea Digital. Revista De Pensamiento E Investigación Social, 1(8). Recuperado de: http://atheneadigital.net/article/view/n8-fernandez/237pdf-es

Fernández, P. (1999). La Afectividad Colectiva. México: Taurus. 
Fernández-Planells, A., Feixa, C. y Figueras, M. (2013). 15-M En España: Diferencias y Similitudes en las Prácticas Comunicativas con los Movimientos Previos. Última década, 21(39), pp. 115-138. Recuperado de: $\quad$ http://www.scielo.cl/scielo.php?script=sci_arttext\&pid=S071822362013000200006

Figueroa, H. (2017). Imaginarios de sujeto en la Era Digital. Pos (identidades) contemporáneas. Quito, Ecuador: Ediciones CIESPAL.

Figueroa, H. (2012). El celular como prótesis móvil en la vida cotidiana de mujeres jóvenes adultas. En Souza, D., Cabello, P. y Del Valle, C. Medios, Edades y Cultura. Chile: Ediciones Universidad de la Frontera, pp. 221-235.

Gergen, K. (2006). El yo saturado, los dilemas de la identidad en la vida contemporánea. Barcelona, España: Editorial Paidós.

González, P. (2014). La utilización de las redes sociales como activismo político en Egipto: La revuelta 2.0 en el contexto de la "Primavera Árabe". La balsa de piedra. Revista de teoría y geoestrategía iberoamericana y mediterránea, $\quad N^{\circ} 8$. España: Universidad Complutense de Madrid.

Gualda, E., Borrero, J. y Carpio, J. (2015). La 'Spanish Revolution' en Twitter (2): Redes de hashtags y actores individuales y colectivos respecto a los desahucios en España. REDES- Revista hispana para el análisis de redes sociales. Vol.26, No.1. España: Universidad Autónoma de Barcelona. ISSN 1579-0185.

Hine, C. (2004): Etnografia virtual. Editorial UOC. Barcelona.

Ibáñez, T. (2004). Introducción a la Psicología Social. Barcelona, España: Editorial UOC.

Ibáñez, T. (2001). Psicología social construccionista (2 $2^{\mathrm{a}}$ Ed.). México: Universidad de Guadalajara.

Iñiguez L. (2003). El Análisis del Discurso en las Ciencias Sociales: variedades, tradiciones y prácticas, Capítulo III en Iñiguez L (Ed) Análisis del discurso. Manual para las Ciencias Sociales. Barcelona: Ediciones UOC. ISBN 8497880008. PP. 83-124.

Juris, J., Pereira, I. y Feixa, C. (2012). La globalización alternativa y los 'novísimos' movimientos sociales. Revista del Centro de Investigación. Universidad La Salle Vol. 10 Núm. 37, pp. 23-39. Recuperado de: http://www.redalyc.org/articulo.oa? $\mathrm{id}=34223328002$

Juris, J. (2012). Reflections on \#Occupy Everywhere: Social Media, Public Space, and Emerging Logics of Aggregation. American Ethnologist $\mathrm{N}^{0} 39$ (2). 
31 Participación social, juventudes, y redes sociales virtuales: rutas transitadas, rutas posibles

Juris, J. y Razsa, M. (2012). Introduction: Occupy, Anthropology and the 2011 Global Uprisings. Cultural Anthropology No27(2).

Lasén, A. y Casado, E. (2014). Mediaciones tecnológicas. Cuerpos, afectos y subjetividades. Madrid: CIS.

Livingstone, S. (2010). Interactivity and participation on the Internet: young people's response to the civic sphere. En Dahlgren, P. (ed.) Young Citizens and New Media: Learning for Democratic Participation. Routledge studies in social and political thought. Routledge, London, UK, pp. 103-124. ISBN 9780415882545

Martín Barbero, J. (1987). De los medios a las mediaciones. Barcelona: Gustavo Gili Ed.

Millaleo, S. (2011). Participación ciudadana on line: Experiencias en el mundo y Chile. En: Girardi, G. Diálogos del Bicentenario del Congreso Nacional, desafios futuros. Chile. Pp. 119-152. Recuperado de: http://www.repositorio.uchile.cl/handle/2250/122724

Montenegro, M. y Pujol, J. (2010). Nuevas tecnologías y agenciamiento juvenil: aproximación desde el ciberfeminismo. Revista de Estudios de Juventud, 89, pp. 221-234.

Mouffe, C. (2007). En torno a lo político. Buenos Aires: Fondo de Cultura Económica.

Morán, M.J. y Benedicto, J. (2008). Las y los jóvenes como actores sociales y políticos en la sociedad global. Revista Bianual Pensamiento Iberoamericano, Temática Inclusión y Ciudadanía: perspectiva de la Juventud en Iberoamérica [online]. No.3, 2da época, pp. 139-164. Disponible

en: http://dialnet.unirioja.es/servlet/articulo?codigo $=2781562$

Palenzuela, Y. (2013). Imaginarios sociales de jóvenes universitarios sobre su participación ciudadana en redes sociales virtuales. Tesis de Magíster en Análisis Sistémico Aplicado a la Sociedad. Facultad de Ciencias Sociales. Universidad de Chile.

Piper, I. (2002). Políticas Sujetos y Resistencias: debates y críticas en Psicología Social. Cuadernos de Psicología Social, Chile: Universidad ARCIS.

Rancière, J. (2009). El reparto de lo sensible. Estética y Política. Chile: LOM Ediciones.

Rancière, J. (2006). Política, Policía, Democracia. Chile: LOM Ediciones.

Reguillo, R. (2017). Paisajes Insurrectos. Ned Ediciones. 
Reguillo, R. (2016). Jóvenes en la encrucijada contemporánea: en busca de un relato de futuro. Debate Feminista, Volumen 48, 2013, pp. 137-151. ISSN 0188-9478. Disponible en: https://ac.elscdn.com/S0188947816300925/1-s2.0-S0188947816300925main.pdf? tid $=864 \mathrm{~b} 42 \mathrm{~d} 8-\mathrm{c} 0 \mathrm{~d} 7-11 \mathrm{e} 7-\mathrm{af} 48-$ 00000aab0f02\&acdnat $=1509741936$ cfc4935d5ce7de808940344fddede $64 \mathrm{~b}$

Reguillo, R. (2007). Emergencias de Culturas Juveniles. Estrategias del desencanto. Colombia: Grupo Editorial Norma.

Retamozo, M. (2009). Lo político y la política: los sujetos políticos, conformación y disputa por el orden social. Revista Mexicana de Ciencias Politicas y Sociales. ISSN 0185-1918. Disponible en: http://www.redalyc.org/articulo.oa?id=42115999004

Rodríguez, I. (2011). Los movimientos sociales como actor-redes: Perspectivas para un enfoque simétrico a las protestas ecologistas de Doñana. Convergencia. Vol.18, n.56, pp. 13-35. ISSN 1405-1435. Disponible en: http://www.scielo.org.mx/pdf/conver/v18n56/v18n56a1.pdf

Roig, A., Sánchez-Navarro, J. y Leibovitz, T. (2012) ¡Esta película la hacemos entre todos! Crowdsourcing y crowdfunding como prácticas colaborativas en la producción audiovisual contemporánea. Icono 14, 10 (1), pp. 25-40.

Roig, A. (2011) La participación como bien de consumo: una aproximación conceptual a las formas de implicación de los usuarios en proyectos audiovisuales colaborativos. Anàlisi: Quaderns de comunicació $i$ cultura, 40, pp. 101-114.

San Cornelio, G. y Cruz, E. G. (2014). Co-creation and Participation as a Means of Innovation in New Media: An Analysis of Creativity in the Photographic Field. International Journal of Communication, 8, 20.

San Cornelio, G., Ardèvol, E., Roig, A., Sánchez-Navarro, J., Gómez-Cruz, E., Morales, J., Ferrer, J., Pagès, R., Leibovitz, T. y Lanzeni. D. (2013). Prácticas creativas y participación en los Nuevos Medios. Informe de investigación relativo al proyecto CREATIVE. Universidad Oberta de Catalunya. Código: HAR2010-18982. Disponible en: https://www.academia.edu/10539215/Informe_final_Pr\%C3\%A1cticas creativas_y_participaci\%C3\%B3n_en_los_nuevos_medios

Sánchez, J. y Torres, R. (2014). Juventud, memoria y movilización en América Latina contemporánea. Santiago: Ril.

Scherman, A. y Arriagada, A. (2010). ¿Ciudadanía digital, participación tradicional? Jóvenes, participación política y consumo de medios en Chile. Ponencia presentada en el III Congreso Latinoamericano de 
33 Participación social, juventudes, y redes sociales virtuales: rutas transitadas, rutas posibles

Opinión Pública, Querétaro, México. Disponible en: http://ebookbrowse.com/scherman-arriagada-pdf-d190579737

Subirat, J. (2015 a). Ya nada será lo mismo: Los efectos del cambio tecnológico en la política, los partidos y el activismo juvenil. Madrid: Ediciones FAD y Centro Reina Sofía sobre Adolescencia y Juventud.

Subirat, J. (2015 b). Todo se mueve. Acción colectiva, acción conectiva. Movimientos, partidos e instituciones. RES N ${ }^{\circ} 24$ pp. 123-131. ISSN: 1578-2824.

Subirats, J. (2011). Otra sociedad. ¿Otra política? De "no nos representan” a la democracia de lo común. Icaria Barcelona.

Torres, R. y Costa, P. (2012). Uso e impacto de las redes sociales de internet sobre las movilizaciones juveniles en Chile: ¿hacia nuevas formas de organización colectiva? En Souza, D.; Cabello, P.; Del Valle, C. Medios, Edades y Cultura. Chile: Ediciones Universidad de la Frontera, pp. 117-138.

Valdettaro, S (2012). Fuego-Revolución-Tecnologías. En La política de los internautas: Nuevas formas de participación. Buenos Aires: Editorial La Crujía, pp. 155 -172.

Valenzuela, S (2012). La protesta en la era de Facebook: Manifestaciones juveniles y uso de redes sociales en Chile 2009-2011. Disponible en: $\mathrm{http} / / /$ academia.edu/1385052/La_protesta_en_la_era_de_Facebook_Ma nifestaciones_juveniles_y_uso_de_redes_sociales_en_Chile_2009-2011

\section{INFORMES Y DOCUMENTOS}

ComScore. (2014). Futuro digital Chile 2014. Disponible en: http://www.comscore.com/lat/Prensa-y-Eventos/Presentaciones-ylibros-blancos/2014/Chile-Digital-Future-in-Focus-2014

INJUV (2013). Séptima Encuesta Nacional de Juventud. Instituto Nacional de Juventud. Gobierno de Chile. Disponible en: http://www.injuv.gob.cl/portal/wpcontent/files_mf/septimaencuestanacionaljuventud2.pdf

INJUV (2010). Sexta Encuesta Nacional de Juventud. Instituto Nacional de Juventud. Gobierno de Chile. Disponible en: $\mathrm{http}: / /$ extranet.injuv.gob.cl/cedoc/encuestasnacionalesdejuventud/SextaE ncuestaNacionaldeJuventud.pdf

INDH (2015). Informe final de resultados III Encuesta Nacional de Derechos Humanos. Instituto Nacional de Derechos Humanos. Santiago de Chile. 
Disponible en:

http://bibliotecadigital.indh.cl/bitstream/handle/123456789/864/Informe. pdf?sequence $=8$

SUBTEL (2013). Chile se mantiene entre los cinco países OCDE de mayor crecimiento en penetración y acceso a banda ancha. Disponible en: $\mathrm{http}: / / \mathrm{www}$. subtel.gob.cl/index.php?option=com_content\&view $=$ article $\& \mathrm{id}=3173 \% 3$ Achile-se-mantiene-entre-los-cinco-paises-ocde-de-mayorcrecimiento-en-penetracion-y-acceso-a-bandaancha\&catid $=93 \% 3 \mathrm{Abanda}-$ ancha\&lang $=\mathrm{es}$

FECHA DE RECEPCIÓN: NOVIEMBRE 2017.

FECHA DE ACEPTACIÓN: DICIEMBRE 2017. 